\title{
Measurement of Total Alkaline Phosphatase Activity in Human Serum using Charcoal Activated Para-Nitro Phenyl Phosphate
}

\author{
Sridhar $\mathbf{K}^{1}$, Narayanan $\mathbf{M}^{2}$, Goswami $\mathbf{K}^{3}$ \\ ${ }^{1,2}$ Meril Diagnostics Pvt. Ltd., Vapi, Gujrat, India \\ ${ }^{3}$ Lincoln University College, Kuala Lumpur, Malaysia
}

\begin{abstract}
A one step Charcoal treated Alkaline phosphatase (ALP) reagent has been developed without affecting its basic ingredients. Conventionally, ALP measurement is based on the detection of phenol produced by an enzymatic reaction in $37^{\circ} \mathrm{C}$ at pH10 using substrate para-nitrophenyl phosphate (pNPP). In present study, measurement range of ALP activity was noted from 6.41 to $628 \mathrm{U} / \mathrm{L}$ in serum samples. Experimental results are compared to these obtained by spectrophotometric measurement in bovine serum. Excellent Correlation exists and close agreements are noted between the both charcoal treated and without charcoal treated reagents having similar substrate (PNPP) properties. Use of charcoal has great advantages in delaying photo degradation of pNPP improving its shelf life \& stability.
\end{abstract}

Keywords: ALP, Charcoal, pNPP, Spectrophotometry, Bovine Serum.

\section{Introduction}

Although introduced more than 50 years ago, the measurement of alkaline phosphatase (ALP, EC 3.1.3.1) activity in serum as an aid in the diagnosis of hepato-biliary and bone disease enjoys continued popularity ${ }^{1}$.Also,alkaline phosphatase comprises a group of enzymes that catalyse the hydrolysis of phosphate esters in an alkaline environment, generating an organic radical and inorganic phosphate ${ }^{2}$.In healthy adults ,this enzyme is mainly derived from from the liver, bones and in lesser amounts from intestine, placenta, kidneys and leucocytes ${ }^{3}$. Several approaches in the determination of alkaline phosphatase activity are commercially employed ${ }^{4}$. The level of alkaline phosphatase activity can be measured and upon enzymatic induced hydrolysis of the substrate para nitro phenyl phosphate (pNPP) in the presence of magnesium ions. These phosphatases hydrolyse pNPP \& ultimately produce end product yellow chromogenic substance para nitrophenoxide, measured at $405 \mathrm{~nm}^{4}$. Considering sensitivity, linearity \& reaction kinetics, P NPP is the ideal choice \& Expert panel on Enzymes of the IFCC have preferred to recommend AMP buffer.

The main objectives of this study is to evaluate the use of Charcoal as a monocomposite package as a new innovative approach for enhanced shelf life of Alkaline phosphatase reagents without affecting enzyme kinetics \& sensitivity.

\section{Methods \& Result}

The principle of method and reagent preparations follows as per guide line of IFCC. The basic properties of the reagent constituents remain same as conventional method excepting addition of Charcoal represented as follows.

2 -Amino-2 methyl-1-propanol $1 \mathrm{~mol} / \mathrm{L}$, 4-Nitrophenyl 1phosphate $1 \mathrm{mmol} / \mathrm{L}$ Magnesium acetate $2.0 \mathrm{mmol} / \mathrm{L}$
Zinc sulfate $1.0 \mathrm{mmol} / \mathrm{L}$

$\mathrm{N}$-(2-Hydroxyethyl) ethylenediaminetri acetic acid (HEDTA) $2 \mathrm{mmol} / \mathrm{L}$

Phenol $1.5 \mathrm{~mol} / \mathrm{L}$

Charcoal $20 \mathrm{mg} / \mathrm{ml}$

$\mathrm{pH}: 10.2 \pm 0.05$

Temperature : $37 \pm 0.1^{\circ} \mathrm{C}$

In the present study, reagent grade charcoal, preferably activated charcoal or active charcoal was used during preparation of conventional reagents for specifically recycling $\mathrm{pNPP}$ and improving stability. The activator charcoal has been treated with oxygen to open up millions of thing pores between the carbon atoms. Background absorbance of Charcoal treated reagent measured photometrically at $405 \mathrm{~nm}$, over a period of 45 days, represented in the following Table 1.

Table 1:

\begin{tabular}{|c|c|c|c|c|}
\hline Days & Batch-1 & Batch-2 & Batch-3 & Batch-4 \\
\hline 0 & 0.065 & 0.056 & 0.067 & 0.075 \\
\hline 10 & 0.097 & 0.089 & 0.102 & 0.110 \\
\hline 30 & 0.115 & 0.121 & 0.118 & 0.135 \\
\hline 45 & 0.131 & 0.132 & 0.136 & 0.143 \\
\hline
\end{tabular}

Comparative studies for serum ALP measurement were undertaken in autoanalyser by using two separate alkaline based (pH10) pNPP reagents having charcoal in one segment and without charcoal in others. In both cases, sample/reagent ratio is $1: 50$, measurement wavelength $405 \mathrm{~nm}$ and it follows Rate principles and type of reaction is positive.

Result shows excellent correlation \& harmonization studies reveals close agreement represented in the following table (Table 2) and (Fig.1 \& 2).

Regression analysis shows positive correlation $\left(\mathrm{R}^{2} 0.982\right)$.

Harmonization studies reveal that there is no appreciable 


\section{International Journal of Science and Research (IJSR) \\ ISSN (Online): 2319-7064 \\ Index Copernicus Value (2013): 6.14 | Impact Factor (2015): 6.391}

change in ALP activity between two sets (p NPP with charcoal \& without charcoal) of reagents. Moreover, distribution of majority values are symmetrical on both sides along the regression line (difference is $\pm 5 \mathrm{U} / \mathrm{L}$ ) against irrespective of difference concentration of ALP activity, excepting in one occasion.

Table 2

\begin{tabular}{|c|c|c|c|}
\hline $\begin{array}{l}\text { pNPPWithout } \\
\text { Charcoal }\end{array}$ & $\begin{array}{l}\text { pNPP with } \\
\text { Charcoal }\end{array}$ & Average & Difference \\
\hline 104 & 103 & 103.5 & -1 \\
\hline 148 & 151 & 149.5 & -3 \\
\hline 48 & 40 & 44 & -8 \\
\hline 75 & 78 & 76.5 & 3 \\
\hline 48 & 40 & 44 & -8 \\
\hline 46 & 41 & 43.5 & -5 \\
\hline 76 & 80 & 78 & 4 \\
\hline 55 & 59 & 57 & 4 \\
\hline 77 & 78 & 77.5 & 1 \\
\hline 52 & 57 & 54.5 & 5 \\
\hline 72 & 69 & 70.5 & -3 \\
\hline 100 & 94 & 97 & -6 \\
\hline 152 & 147 & 149.5 & -5 \\
\hline 102 & 105 & 103.5 & 3 \\
\hline 47 & 39 & 43 & -8 \\
\hline 46 & 40 & 43 & -6 \\
\hline 61 & 60 & 60.5 & -1 \\
\hline 59 & 58 & 58.5 & -1 \\
\hline 60 & 60 & 60 & 0 \\
\hline 102 & 100 & 101 & -2 \\
\hline 78 & 80 & 79 & 2 \\
\hline 61 & 60 & 60.5 & -1 \\
\hline 75 & 78 & 76.5 & 3 \\
\hline 65 & 61 & 63 & -4 \\
\hline 45 & 39 & 42 & -6 \\
\hline
\end{tabular}

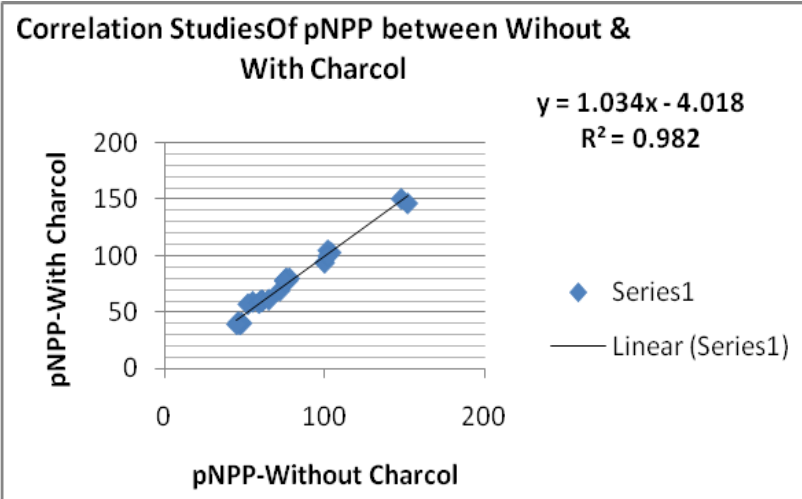

Figure 1

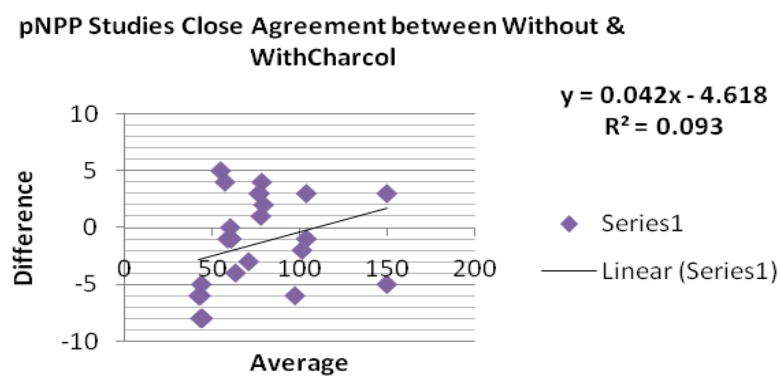

Figure 2
Precision Check: Harmonization studies of ALP activity were under taken in 2 different throughput auto analyzers (100 tests / hour \& 400 tests / hour) for precision checking of Charcoal based pNPP reagents represented in the following Table 3 \& Fig. 3 \& 4 .

Table 3

\begin{tabular}{|c|c|c|c|}
\hline AUTOQUANT-100 & AUTOQUANT-400 & Average & Difference \\
\hline 57.91 & 56.8 & 57.355 & 1.11 \\
\hline 71.27 & 72.51 & 71.89 & -1.24 \\
\hline 72.99 & 75.61 & 74.3 & -2.62 \\
\hline 51.59 & 50.2 & 50.895 & 1.39 \\
\hline 58.28 & 61.3 & 59.79 & -3.02 \\
\hline 81.29 & 76.42 & 78.855 & 4.87 \\
\hline 65.33 & 63.24 & 64.285 & 2.09 \\
\hline 80.55 & 79.2 & 79.875 & 1.35 \\
\hline 77.21 & 76.41 & 76.81 & 0.8 \\
\hline 53.45 & 54.02 & 53.735 & -0.57 \\
\hline 113.95 & 114.7 & 114.325 & -0.75 \\
\hline 104.3 & 95.67 & 99.985 & 8.63 \\
\hline 61.25 & 58.93 & 60.09 & 2.32 \\
\hline 96.68 & 97.13 & 96.905 & -0.45 \\
\hline 68.3 & 68.25 & 68.275 & 0.05 \\
\hline 77.58 & 79.3 & 78.44 & -1.72 \\
\hline 66.44 & 65.12 & 65.78 & 1.32 \\
\hline 90.94 & 88.2 & 89.57 & 2.74 \\
\hline 79.06 & 81.2 & 80.13 & -2.14 \\
\hline 44.54 & 48.6 & 46.57 & -4.06 \\
\hline & & & \\
\hline
\end{tabular}

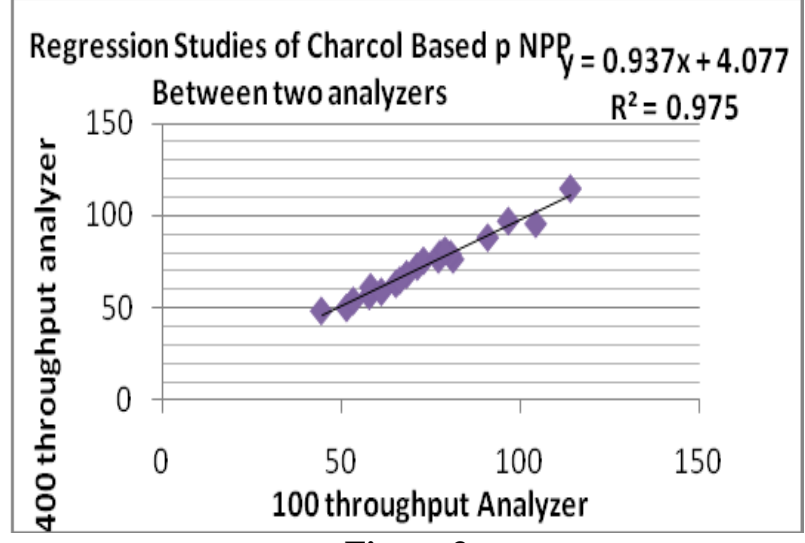

Figure 3

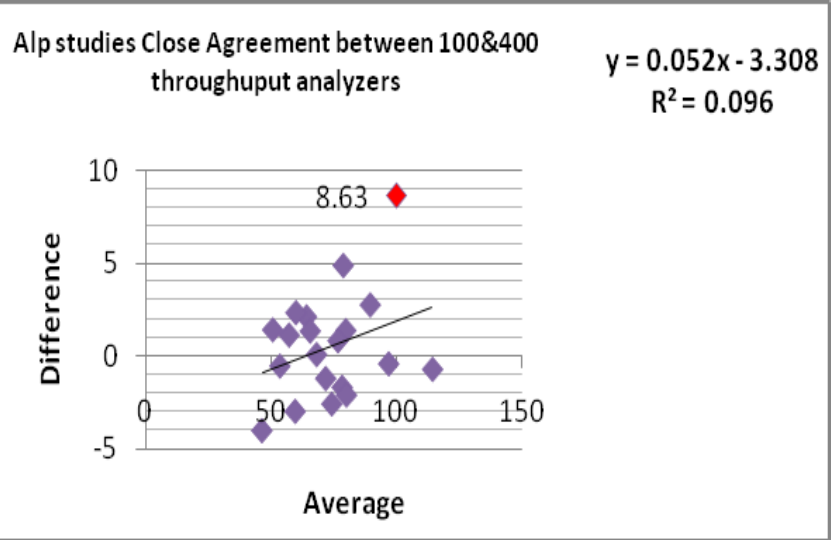

Figure 4

Table 3 \& Fig. 3 \& 4 demonstrate that ALP activity is strongly precise between the two different throughput analyzers by using Charcoal based pNPP as it is evident by 


\section{International Journal of Science and Research (IJSR) \\ ISSN (Online): 2319-7064 \\ Index Copernicus Value (2013): 6.14 | Impact Factor (2015): 6.391}

both regression analysis ( $\mathrm{R}^{2}$ 0.975) and close agreement studies. Close agreement studies reveals that in majority cases, the difference of ALP activity (Y axis) is within \pm 5 $\mathrm{U} / \mathrm{L}$ against irrespective of different conc. of ALP activity (X-axis), excepting in one occasion, the difference of ALP activity is 8.63. In majority cases, the data points are symmetrically distributed along the close proximity of the regression line.

\section{Sensitivity \& Linearity Check}

Linearity check was carried out by selecting high reacting serum samples having ALP activity 628 U/L. Sample was serially diluted up to different concentration. Correlation studies were conducted by measuring individual samples having respective concentration and its corresponding to observed response.

Table 5

\begin{tabular}{|c|c|}
\hline Serial dilutions concentration of ALP & Observed Response \\
\hline 628 & 628 \\
\hline 314 & 348.3 \\
\hline 157 & 167.7 \\
\hline 78.5 & 83.64 \\
\hline 39.25 & 40 \\
\hline 19.6 & 17.83 \\
\hline 9.81 & 8.9 \\
\hline 4.9 & 6.41 \\
\hline
\end{tabular}

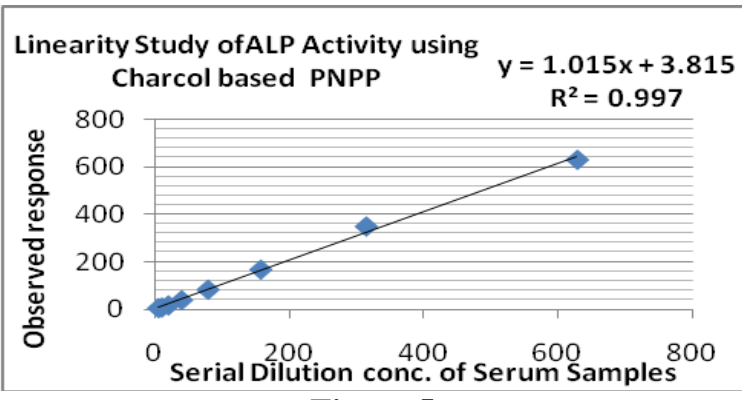

Figure 5

Result shows (Table 5 \& Fig. 5) excellent correlation $\left(\mathrm{R}^{2}\right.$ 0.997) and minimum activity $6.41 \mathrm{U} / \mathrm{L}$ was noted.

\section{Summary \& Conclusion}

The present study reveals that there is no appreciable changes in background absorbance of charcoal treated p NPP over a period of 45 days (Table-1). It is plausible to note that, Alkaline Phpsphatase activity remains consistent both in Charcoal treated p NPP reagents \& p NPP reagents having without charcoal as it is reflected in Table 2, Fig. 1 \& Fig. 2 Precision (Table 3, Fig. 3 \& 4) and Linearity (Table 5, Fig. 5) studies reveals that Charcoal based pNPP reagents maintains persistent ALP activity without compromise statistical rules.

In conclusion, Charcoal based $\mathrm{p}$ NPP reagents can be alternative choice for ALP measurement having slower rate of photo degradation, improving stability, minimizes reagent wastage and savings operating cost.

\section{Acknowledgement}

The authors are grateful to Meril Diagnostics Pvt. Ltd. for giving laboratory facilities and kind permission to publish this paper.

\section{References}

[1] Mc. Comb RB, Bowers GN Jn. ,Posen S. Alkaline Phosphatase, Plenum Press, New York,NY,1979

[2] Reichnling JJ, Kaplan MM, Clinical use of serum enzymes in liver diseases. Dia.Dis.Sci.1988,33:1601-1614

[3] Mc. Intyre N. Rosallin S, Biochemical investigation in the management of liver disease. In Oxford University Press,1991,293-309

[4] Tietz NW, Bootis EA, et.al. A reference method for measurement of alkaline phosphatase activity in human serum. Clin.Chem 29/5,751-761(1983).

[5] Expert panel on enzymes, committee on standards (IFCC).Approved recommendation of IFCC methods for the measurement of catalytic concentration of enzymes. Part 1.General consideration Clin.Chem Acta.98, 163F-174F (1979). 